\title{
Cluster algorithm for Potts models with fixed spin densities
}

\author{
R. P. Bikker and G. T. Barkema \\ Theoretical Physics, Utrecht University, Princetonplein 5, 3584 CC Utrecht, The Netherlands
}

(Received 3 May 2000; revised manuscript received 16 June 2000)

\begin{abstract}
A cluster algorithm is presented for the simulation of the $q$-state Potts models in which the number of spins is conserved in each state. The algorithm constructs Fortuin-Kasteleyn cluster configurations from spin configurations, in a way identical to the Swendsen-Wang algorithm; the spin assignment to these clusters is, however, different, and conserves the number of spins for each state. Compared to traditional nonlocal spinexchange algorithms, the cluster algorithm presented here suffers less from critical slowing down, and consequently is more efficient near the critical temperature.
\end{abstract}

PACS number(s): 02.70.Lq, 05.50.+q, 64.60.Ht, 75.10.Hk

\section{INTRODUCTION}

Before 1987, the Potts model was almost exclusively simulated by means of the Metropolis algorithm [1], in which single-spin updates are proposed and either accepted or rejected depending on the change in energy. This algorithm works quite satisfactorily, except close to the critical point. At the critical temperature, the correlation times increase with system size as $L^{z}$, with a critical dynamic exponent equal to or slightly above 2: for the two-state Potts model (Ising model), the critical dynamical exponent is reported to be $z=2.167 \pm 0.001$ in two and $z=2.02 \pm 0.02$ in three dimensions [2]. The introduction of cluster algorithms has greatly advanced the accuracy with which critical properties of the Potts model and many other models in statistical physics can be studied. The first widely used cluster algorithm was introduced by Swendsen and Wang [3,4]; we will describe their algorithm in Sec. II A. The dynamic exponent of the Swendsen-Wang algorithm in the two- and threedimensional Ising model is reported to be $z=0.25 \pm 0.01$ and $z=0.54 \pm 0.02$, respectively [5], while for the twodimensional three-state Potts model, a dynamical critical exponent of $z=0.515 \pm 0.002$ is reported [6]: cluster algorithms are able to significantly reduce critical slowing down.

To study multicomponent lattice gases in the coexistence regime, for instance to study interfaces or equilibrium crystal shapes, one has to fix the number of particles in the lattice gas for each component, i.e., the spin density for each state. One typically resorts to spin-exchange dynamics, with the unfortunate consequence of a critical slowing down at least as severe as experienced with the Metropolis algorithm applied to the regular Potts model. The usual cluster algorithms do not conserve the spin densities. For the conserved-orderparameter Ising model, Heringa and Blöte $[7,8]$ recently introduced a cluster algorithm, which is in spirit related to the Wolff algorithm $[9,4]$. It is reported to have hardly any critical slowing down, with a dynamical exponent of $z=0.21$. This algorithm has not been generalized to Potts models with more than two states.

In this paper, we present a modification of the SwendsenWang algorithm, to conserve the spin densities. In the following section, we describe their algorithm, and introduce our modified density-conserving cluster algorithm. In Sec. III, we present measurements of the critical dynamic expo- nent for our algorithm, and show its efficiency. The paper is concluded with a summary and conclusions, and a discussion of future work.

\section{CLUSTER ALGORITHMS}

\section{A. The Swendsen-Wang algorithm}

The Swendsen-Wang algorithm is designed to simulate the Potts model, defined by the Hamiltonian

$$
H=-J \sum_{\langle i, j\rangle} \delta\left(\sigma_{i}, \sigma_{j}\right)
$$

in which $J$ is the coupling constant, $\delta$ denotes the Kronecker delta function, and the summation runs over all pairs of nearest-neighbor sites, each having a spin with value $(\sigma$ $=1, \ldots, Q)$. We use the usual symbols $N$ for the number of lattice sites, $L$ for the lateral dimension of the lattice with periodic boundary conditions, and $\rho_{i}=(1 / N) \Sigma_{k} \delta\left(\sigma_{i}, k\right)$ for the density of spins with value $i$. The number of different spin values $Q$ can have any integer value.

In this algorithm, the entire lattice is divided into clusters of aligned spins, to each of which a random new value is assigned. In detail, one step of the algorithm proceeds as follows. (i) Visit all nearest-neighbor pairs of lattice sites; do nothing if the two spins are not aligned, but if they are, activate the bond between those two sites with a probability $P_{c}=1-\exp (-\beta J)$, where $\beta$ is the inverse temperature. (ii) Group lattice sites that are connected by such activated bonds into clusters. (iii) Select a random new spin value for each cluster, and assign this spin value to each of the sites constituting the cluster. Steps (i)-(iii) are to be repeated many times, to obtain a set of sample configurations.

The proof of correctness for our density-conserving cluster algorithm is based on that for the Swendsen-Wang algorithm, which is presented in the remainder of this section. First we show detailed balance, next we discuss ergodicity.

Suppose we denote the spin configuration before and after the move by $C_{a}$ and $C_{b}$, respectively, with total energies $E_{a}$ and $E_{b}$, and the intermediate cluster configuration $C_{m}$ (also known as the Fortuin-Kasteleyn representation [10]); furthermore, we write the probability to move from a configuration $X$ to configuration $Y$ as $T(X \rightarrow Y)$. Then, the probability to move from a spin configuration $C_{a}$ to a cluster configuration 
$C_{m}$ is a product with factors $P_{c}$ over all nearest-neighbor pairs of spins that are connected, times a product with factors $1-P_{c}$ over all aligned nearest-neighbor pairs of spins that are disconnected:

$$
\begin{aligned}
& T\left(C_{a} \rightarrow C_{m}\right)=\prod_{\langle i, j\rangle}\left(P_{c}\right) \quad \prod_{\langle i, j\rangle}\left(1-P_{c}\right) \\
& \sigma_{i}^{(a)}=\sigma_{j}^{(a)} \quad \sigma_{i}^{(a)}=\sigma_{j}^{(a)} \\
& i, j \text { conn. } i, j \text { disconn. }
\end{aligned}
$$

and a similar expression for $T\left(C_{b} \rightarrow C_{m}\right)$. Since spins that are connected are necessarily aligned both before and after the move, the first product on the right-hand side is equal in $T\left(C_{a} \rightarrow C_{m}\right)$ and $T\left(C_{b} \rightarrow C_{m}\right)$. All factors in the second product on the right-hand side dealing with pairs of spins that are aligned both before and after the move are also equal in $T\left(C_{a} \rightarrow C_{m}\right)$ and $T\left(C_{b} \rightarrow C_{m}\right)$. That leaves in the ratio of the transition rates only the factors dealing with disconnected pairs of spins that are aligned either in configuration $C_{a}$ or in configuration $C_{b}$, but not both. The ratio of the transition rates $T\left(C_{a} \rightarrow C_{m}\right)$ and $T\left(C_{b} \rightarrow C_{m}\right)$ therefore reduces to

$$
\begin{aligned}
& \frac{T\left(C_{a} \rightarrow C_{m}\right)}{T\left(C_{b} \rightarrow C_{m}\right)}=\prod_{\langle i, j\rangle}\left(1-P_{c}\right) / \prod_{\langle i, j\rangle}\left(1-P_{c}\right) . \\
& \sigma_{i}^{(a)}=\sigma_{j}^{(a)} \quad \sigma_{i}^{(a)} \neq \sigma_{j}^{(a)} \\
& \sigma_{i}^{(b)} \neq \sigma_{j}^{(b)} \quad \sigma_{i}^{(b)}=\sigma_{j}^{(b)}
\end{aligned}
$$

Using that $\ln \left(1-P_{c}\right)=-\beta J$, in combination with some rewriting, we obtain for the logarithm of this ratio

$$
\begin{aligned}
& \ln \left(T\left(C_{a} \rightarrow C_{m}\right)\right)-\ln \left(T\left(C_{b} \rightarrow C_{m}\right)\right) \\
& \quad=-\beta J \sum_{\langle i, j\rangle}\left[\delta\left(\sigma_{i}^{(a)}, \sigma_{j}^{(a)}\right)-\delta\left(\sigma_{i}^{(b)}, \sigma_{j}^{(b)}\right)\right] .
\end{aligned}
$$

As can easily been seen from the Hamiltonian Eq. (1), this is equal to $\beta\left(E_{a}-E_{b}\right)$. Since $T\left(C_{m} \rightarrow C_{a}\right)=T\left(C_{m} \rightarrow C_{b}\right)$ $=2^{-n}$, where $n$ is the number of clusters in $C_{m}$, detailed balance follows:

$$
\begin{gathered}
\frac{T\left(C_{b} \rightarrow C_{a}\right)}{T\left(C_{a} \rightarrow C_{b}\right)}=\frac{T\left(C_{b} \rightarrow C_{m}\right) T\left(C_{m} \rightarrow C_{a}\right)}{T\left(C_{a} \rightarrow C_{m}\right) T\left(C_{m} \rightarrow C_{b}\right)} \\
=\exp \left(-\beta\left(E_{a}-E_{b}\right)\right) .
\end{gathered}
$$

In addition to obeying detailed balance, the algorithm is ergodic, since there is a finite probability that in a given move all clusters will contain one site only, to which any value can be assigned. Since this algorithm is ergodic and satisfies detailed balance, it is guaranteed that eventually these sample configurations will be drawn from the Boltzmann distribution for the regular Potts model. The densities $\rho_{i}$ are not conserved in the Swendsen-Wang algorithm.

\section{B. Density-conserving cluster algorithm}

The topic of this paper is to present a modification to this algorithm that ensures the conservation of the densities. This modification is made in step (iii), in which the new spin values are assigned: rather than assigning random spin val- ues to each cluster, we redistribute spin values over the clusters while conserving the spin densities. As for the original Swendsen-Wang algorithm, the general idea is a two-step approach, $C_{a} \rightarrow C_{m} \rightarrow C_{b}$, where all the energetics required for obtaining detailed balance are incorporated in the construction of the clusters, and detailed balance is achieved by conservation of the property $T\left(C_{m} \rightarrow C_{a}\right)=T\left(C_{m} \rightarrow C_{b}\right)$.

The first step towards such an algorithm is to devise an elementary move. The move we are looking for is identifying one set of aligned clusters with spin value $q_{1}$ and another such set with spin value $q_{2} \neq q_{1}$ with exactly the same area (number of sites), and then exchanging the spin values $q_{1}$ and $q_{2}$.

How do we identify such sets? First of all, for each spin value $i=1, \ldots, Q$ we group all clusters with spin value $i$ into the set $S_{i}$. Next, within each set we list these clusters in random order, and keep track of the cumulative area. Every time that in two sets the same value for the cumulative area occurs, we have found an exchange point. If the spin values are exchanged in all clusters up to the exchange point, while the original spin values in all other clusters are conserved, the spin values of two sets of clusters are exchanged without violation of the spin-density conservation (see Fig. 1).

Unless extra measurements are taken, an algorithm based on these elementary moves will not obey detailed balance: the probability of the occurrence of an exchange point is not necessarily equal before and after the cluster exchange. We denote the total number of clusters with spin value $q$ before the exchange takes place as $n_{q}$. Suppose that the exchange takes place between clusters with spin 1 and 2 , and that the number of clusters with spin 1 and 2 that are to be exchanged is $a_{1}$ and $a_{2}$, respectively, while the number of clusters with spin 1 and 2 that are not to be exchanged is $n_{1}$ $-a_{1}$ and $n_{2}-a_{2}$, respectively. The likelihood that there is an exchange point exactly between these sets of clusters is then equal to

$$
T(\rightarrow)=\left[\left(\begin{array}{l}
n_{1} \\
a_{1}
\end{array}\right)\left(\begin{array}{l}
n_{2} \\
a_{2}
\end{array}\right)\right]^{-1},
$$

while after the exchange this probability becomes

$$
\begin{aligned}
T(\rightarrow) & =\left[\left(\begin{array}{l}
n_{1}^{\prime} \\
a_{2}
\end{array}\right)\left(\begin{array}{l}
n_{2}^{\prime} \\
a_{1}
\end{array}\right)\right]^{-1} \\
& =\left[\left(\begin{array}{c}
a_{2}+n_{1}-a_{1} \\
a_{2}
\end{array}\right)\left(\begin{array}{c}
a_{1}+n_{2}-a_{2} \\
a_{1}^{-1}
\end{array}\right)\right]^{-1} .
\end{aligned}
$$

To restore detailed balance, it suffices to introduce a $\mathrm{Me}$ tropolis acceptance ratio:

$$
P_{a}=\min \left[1, \frac{n_{1}^{\prime} ! n_{2}^{\prime} !}{n_{1} ! n_{2} !}\right] .
$$

Once this acceptance probability is included, the elementary move can be used for a correct algorithm, since for two configurations $X$ and $Y$, we now restored the property $T\left(C_{m} \rightarrow X\right)=T\left(C_{m} \rightarrow Y\right)$. 


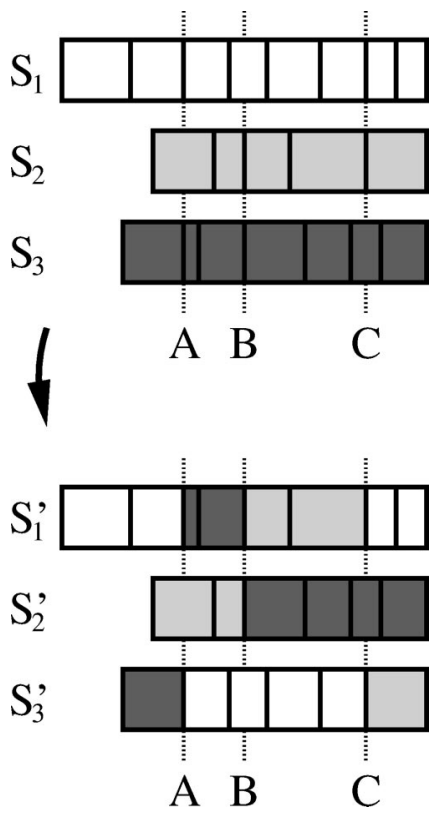

FIG. 1. Assignment of new spin values to the clusters in the three-state Potts model. The upper and lower part are the situations before and after cluster assignment, respectively. The length of each bar corresponds to the total mass $\rho_{i} N$ in the set $S_{i}$. The different shades indicate different spin values before the assignment. The thick lines separate subsequent clusters in each list. The dotted lines indicate the exchange points $A, B$, and $C$, where two of the masses coincide and after which the corresponding spin values are exchanged with the probability given in Eq. (8).

In an actual implementation, the total procedure is to make for each spin value a cumulative list of clusters, where the clusters are placed in random order. Next, all exchange points are identified; the corresponding exchanges are accepted with the probability as given in Eq. (8). It can be verified that also for the concatenation of exchange points, the product over all exchange points of the ratio of forward and backward acceptance probabilities, as given in Eq. (8), equals

$$
\prod_{q=1}^{Q}\left(n_{q}^{\prime} !\right) / \prod_{q=1}^{Q}\left(n_{q} !\right)
$$

which exactly cancels the ratio of the number of ways in which the clusters can be sorted, i.e., the ratio of selection probabilities in the forward and backward direction. Consequently, $T\left(C_{m} \rightarrow C_{a}\right)=T\left(C_{m} \rightarrow C_{b}\right)$.

The density-conserving algorithm is ergodic for the same reason that the Swendsen-Wang algorithm is ergodic: there is a finite probability that all clusters contain one site only, and then each of these can obtain any spin value (under the constraint on the densities).

Having shown that the basic steps of our algorithm are correct, we will now summarize the procedure in the form of a stepwise algorithm. Steps number (i) and (ii) of the Swendsen-Wang algorithm remain unchanged. Step (iii) becomes the following. (iiia) For each state $q \in\{1, \ldots, Q\}$, list all clusters with this spin value in list $S_{q}$ in random order. (iiib) Order the lists with respect to the total area of their not-yet-assigned clusters. Use a random order for lists with

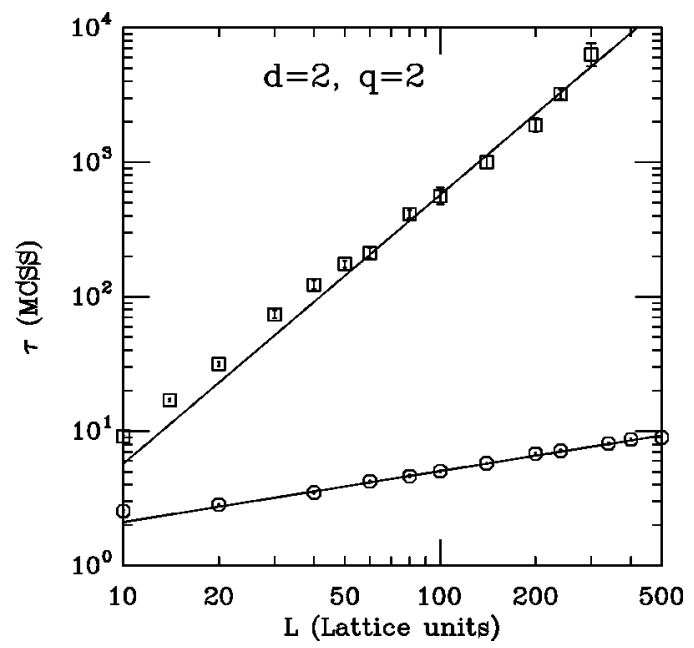

FIG. 2. Correlation time $\tau$ as a function of linear system size $L$ for the two-dimensional two-state Potts model, for spin-exchange dynamics (squares) and the magnetization-conserving cluster algorithm (circles). The lines have exponents of $z=2$ and $z=0.38$.

equal such areas. If the first two lists (those with the largest and next-largest areas) are equal, exchange their colors with the probability as given by Eq. (8). Select one cluster from the first list and assign to it a new color. Update the ordering and repeat this step until spin values are assigned to all clusters.

Note that the computational effort required for step (iii) scales with the total number of clusters $n=\Sigma_{q} n_{q}$, whereas step (ii) scales with the number of spins $N$ in the system. Since $n \ll N$, step (iii) is repeated $N /(2 n)$ times for each time step (ii) is performed, and we still have an implementation in which the computational effort per sweep scales linearly with the number of sites; this greatly decreases the autocorrelation time. It actually also reduces the dynamical critical exponent $z$, since the ratio $N / n$ varies with the system size.

\section{COMPUTATIONAL PROPERTIES}

In order to compare the efficiency of the densityconserving cluster algorithm presented above with that of nonlocal spin-exchange (Kawasaki [11]) dynamics, we have computed the energy autocorrelation times in both the twoand the three-state Potts models, at critical temperature and equal spin densities, for several system sizes. For all data points the correlation time $\tau$ was obtained from a leastsquares fit of the form $e^{-t / \tau}$, to the energy autocorrelation function. For the spin-exchange algorithm, these fits were done in the region where the autocorrelation drops from $e^{-1}$ to $e^{-2}$; for the cluster algorithm, we fitted in a broader region (from 1 to $e^{-3}$ ), in order to have enough points to fit. Time is measured in Monte Carlo steps per site (MCSS); for the cluster algorithm, one MCSS is equal to one lattice update. All runs where started from a random configuration, which was thermalized over a time varying from 6000 to 60000 MCSS. In order to generate enough statistics, the total length of the runs was set to ten times the thermalization time. The statistical errors were determined by repeating each run 10 to 50 times.

Figures 2 and 3 show the correlation times as a function of the linear system size $L$ of the two- and three-dimensional 


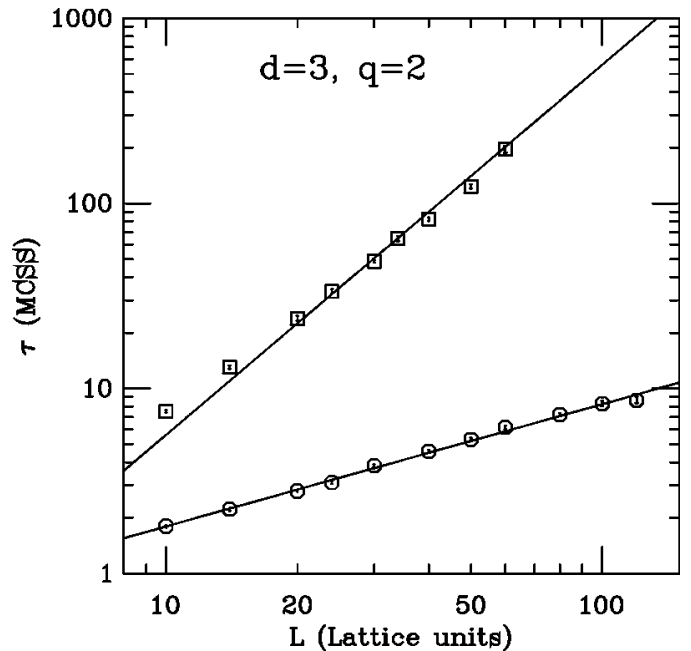

FIG. 3. Correlation time $\tau$ as a function of linear system size $L$ for the three-dimensional two-state Potts model, for spin-exchange dynamics (squares) and the magnetization-conserving cluster algorithm (circles). The lines have exponents of $z=2$ and $z=0.66$.

two-state Potts model, respectively.

For the two-state Potts model, using the nonlocal spinexchange algorithm, we find a critical dynamic exponent of $z=2.0$ in both two and three dimensions. This is in good agreement with the exponents of the three-dimensional nonconserving Metropolis algorithm $(z=2.02 \pm 0.02)$ but not with the critical exponent for the two-dimensional nonconserving Metropolis algorithm $(z=2.167 \pm 0.001)$. Perhaps this is an indication that the conservation of the order parameter affects the critical dynamical exponent, but our statistics are not conclusive. Using our new density-conserving cluster algorithm for the two-state Potts model, we find values for the critical dynamic exponent of $z=0.38 \pm 0.01$ in two and $z=0.66 \pm 0.02$ in three dimensions. These values are both slightly larger than nonconserved Swendsen-Wang values ( $z=0.25 \pm 0.01$ and $z=0.54 \pm 0.02$ for two and three dimensions, respectively).

Figures 4 and 5 show the correlation times as a function

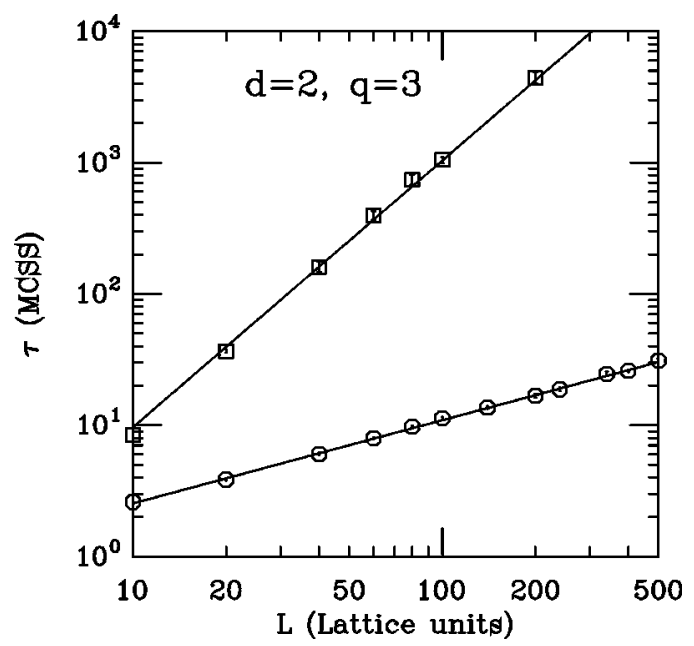

FIG. 4. Correlation time $\tau$ as a function of linear system size $L$ for the two-dimensional three-state Potts model, for spin-exchange dynamics (squares) and the magnetization-conserving cluster algorithm (circles). The lines have exponents of $z=2$ and $z=0.63$.

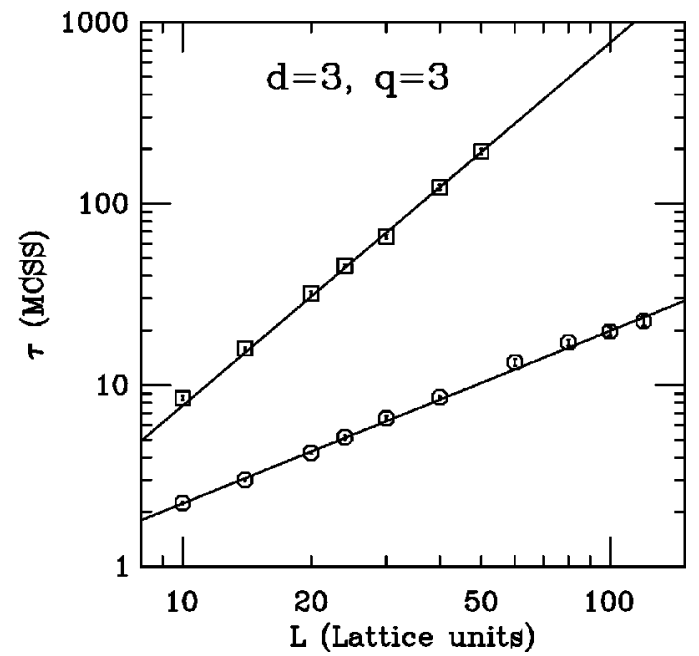

FIG. 5. Correlation time $\tau$ as a function of linear system size $L$ for the three-dimensional three-state Potts model, for spin-exchange dynamics (squares) and the magnetization-conserving cluster algorithm (circles). The lines have exponents of $z=2$ and $z=0.95$.

of the linear system size $L$ of the two- and three-dimensional three-state Potts model, respectively. For the three-state Potts model, using the Metropolis algorithm we find $z=2.0$ in both two and three dimensions. Using our cluster algorithm we find values of $z=0.63 \pm 0.01$ in two and $z=0.95 \pm 0.02$ in three dimensions.

As expected, we find that the cluster algorithm suffers significantly less from critical slowing down and clearly outperforms spin-exchange dynamics at physically interesting lattice sizes in both two and three dimensions and in both the Ising model and the three-state Potts model. Since one move in our cluster algorithm takes an amount of CPU time comparable to what is required for one sweep in the nonlocal spin exchange, our cluster algorithm outperforms nonlocal spin exchange by one or two orders of magnitude, depending on the system size.

\section{SUMMARY AND FUTURE WORK}

We have presented a density-conserving cluster algorithm for the Potts model. This algorithm is only moderately sensitive to critical slowing down: its dynamic critical exponent is found to be $z=0.38 \pm 0.01$ and $z=0.66 \pm 0.02$ for the twoand three-dimensional Ising model, respectively, and $z$ $=0.63 \pm 0.01$ and $z=0.95 \pm 0.02$ for the two- and threedimensional three-state Potts model, respectively. It outperforms the traditional algorithm, nonlocal spin exchange, by one or two orders of magnitude.

In future research, we will use this algorithm to study wetting properties, where the wetting takes place at a curved interface between two coexisting phases; such nonflat interfaces arise, for instance, between a droplet and a surrounding fluid. Other future applications will include the study of line tension between three coexisting phases, and equilibrium shapes in multicomponent mixtures.

\section{ACKNOWLEDGMENTS}

Useful discussions with Henk van Beijeren and Matthieu Ernst are gratefully acknowledged. 
[1] N. Metropolis, A.W. Rosenbluth, M.N. Rosenbluth, A.H. Teller, and E. Teller, J. Chem. Phys. 21, 1087 (1953).

[2] M.P. Nightingale and H.W.J. Blöte, Phys. Rev. Lett. 76, 4548 (1996)

[3] R.H. Swendsen and J.-S. Wang, Phys. Rev. Lett. 58, 86 (1987).

[4] M.E.J. Newman and G.T. Barkema, Monte Carlo Methods in Statistical Physics (Oxford University Press, Oxford, 1999).

[5] P.D. Coddington and C.F. Baillie, Phys. Rev. Lett. 68, 962
(1992).

[6] J. Salas and A.D. Sokal, J. Stat. Phys. 87, 1 (1997).

[7] J.R. Heringa and H.W.J. Blöte, Phys. Rev. E 57, 4976 (1998).

[8] J.R. Heringa and H.W.J. Blöte, Physica A 254, 156 (1998).

[9] U. Wolff, Phys. Rev. Lett. 62, 361 (1989).

[10] C.M. Fortuin and P.W. Kasteleyn, Physica (Amsterdam) 57, 536 (1972).

[11] K. Kawasaki, Phys. Rev. 145, 224 (1966). 